\title{
Effect of quenched disorder on the quantum spin liquid state of the triangular-lattice antiferromagnet $1 T-\mathrm{TaS}_{2}$
}

\author{
H. Murayama, ${ }^{1}$ Y. Sato, ${ }^{1}$ T. Taniguchi, ${ }^{1}$ R. Kurihara, ${ }^{1}$ X. Z. Xing, ${ }^{1}$ W. Huang, ${ }^{1}$ S. Kasahara,,${ }^{1}$ Y. Kasahara, ${ }^{1}$ I. Kimchi, ${ }^{2}$ \\ M. Yoshida, ${ }^{3}$ Y. Iwasa, ${ }^{3,4}$ Y. Mizukami,${ }^{5}$ T. Shibauchi, ${ }^{5}$ M. Konczykowski, ${ }^{6}$ and Y. Matsuda ${ }^{1}$ \\ ${ }^{1}$ Department of Physics, Kyoto University, Kyoto 606-8502, Japan \\ ${ }^{2}$ JILA, NIST, and Department of Physics, University of Colorado, Boulder, Colorado, USA \\ ${ }^{3}$ RIKEN Center for Emergent Matter Science (CEMS), Wako 351-0198, Japan \\ ${ }^{4}$ Quantum-Phase Electronics Center and Department of Applied Physics, University of Tokyo, Tokyo 113-8656, Japan \\ ${ }^{5}$ Department of Advanced Materials Science, University of Tokyo, Chiba 277-8561, Japan \\ ${ }^{6}$ Laboratoire des Solides irradiées, CEA/DRF/IRAMIS, Ecole Polytechnique, CNRS, Institut Polytechnique de Paris, \\ F-91128 Palaiseau, France
}

(Received 16 March 2018; revised manuscript received 2 December 2019; published 29 January 2020)

\begin{abstract}
A quantum spin liquid (QSL) is an exotic state of matter characterized by quantum entanglement and the absence of any broken symmetry. A longstanding open problem, which is key for fundamental understanding the mysterious QSL states, is how the quantum fluctuations respond to randomness due to quenched disorder. Transition metal dichalcogenide $1 \mathrm{~T}-\mathrm{TaS}_{2}$ is a candidate material that hosts a QSL ground state with spin- $1 / 2$ on the two-dimensional perfect triangular lattice. Here, we performed systematic studies of low-temperature heat capacity and thermal conductivity on pure, Se-substituted, and electron-irradiated crystals of $1 \mathrm{~T}-\mathrm{TaS}_{2}$, where the substitution of $\mathrm{S}$ by Se induces weak disorder and electron irradiation induces strong quenched disorder. In pure $1 \mathrm{~T}-\mathrm{TaS}_{2}$, the linear temperature term of the heat capacity $\gamma T$ and the finite residual linear term of the thermal conductivity in the zero-temperature limit $\kappa_{0} / T \equiv \kappa / T(T \rightarrow 0)$ are clearly resolved, consistent with the presence of gapless spinons with a Fermi surface. Moreover, while the strong magnetic field slightly enhances $\kappa_{0} / T$, it strongly suppresses $\gamma$. These unusual contrasting responses to magnetic field imply the coexistence of two types of gapless excitations with itinerant and localized characters. Introduction of additional weak random exchange disorder in $1 \mathrm{~T}-\mathrm{Ta}\left(\mathrm{S}_{1-x} \mathrm{Se}_{x}\right)_{2}$ leads to vanishing of $\kappa_{0} / T$, indicating that the itinerant gapless excitations are sensitive to the disorder. On the other hand, in both pure and Se-substituted systems, the magnetic contribution of the heat capacity obeys a universal scaling relation, which is consistent with a theory that assumes the presence of localized orphan spins forming random singlets. These results appear to capture an essential feature of the QSL state of $1 \mathrm{~T}-\mathrm{TaS}_{2}$; localized orphan spins induced by disorder form random valence bonds and are surrounded by a QSL phase with spinon Fermi surface. Electron irradiation in pure 1T-TaS 2 largely enhances $\gamma$ and changes the scaling function dramatically, suggesting a possible new state of spin liquid.
\end{abstract}

DOI: 10.1103/PhysRevResearch.2.013099

\section{INTRODUCTION}

A spin liquid is a state of matter in which the spins are correlated yet fluctuate strongly, like in a liquid, down to very low temperatures. In particular, a quantum spin liquid (QSL) is a state in which enhanced quantum fluctuations prevent the system from long-range magnetic ordering even at zero temperature [1,2], forming a complex superposition of singlet states. Since these states never order, they have no broken symmetries and are not described by Landau's theory of phase transitions. The ground states of the QSLs are quantum mechanically entangled and are expected to host

Published by the American Physical Society under the terms of the Creative Commons Attribution 4.0 International license. Further distribution of this work must maintain attribution to the author(s) and the published article's title, journal citation, and DOI. fractional quasiparticle excitations. The notion of the QSLs is firmly established in one-dimensional (1D) spin systems as well as in their ladder cousins. In two-dimensional (2D) and three-dimensional (3D) systems, on the other hand, it is widely believed that QSLs are usually realized in the presence of competing orders or geometrical frustrations. Despite tremendous efforts during the past several decades, however, the true nature of the ground states of QSL candidate materials has still remained elusive. One of the most important and longstanding open problems, which is key for understanding the mysterious QSL states both theoretically and experimentally, is how the quantum fluctuations respond to local randomness induced by quenched disorder, such as defects and impurities.

Of specific interest in 2D frustrated spin systems has been the quantum spin-1/2 triangular-lattice Heisenberg antiferromagnet, which has been suggested to be a prototype of a QSL in the resonating valence bond (RVB) model with a quantum superposition of spin singlets $[3,4]$. 
Unfortunately, only a few candidates of the QSL states have been reported for $2 \mathrm{D}$ triangular lattice, including organic Mott insulators, $\kappa$-(BEDT-TTF) ${ }_{2} \mathrm{Cu}_{2}(\mathrm{CN})_{3}[5,6]$ (hereafter abbreviated as BEDT-TTF), $\mathrm{EtMe}_{3} \mathrm{Sb}\left[\mathrm{Pd}(\mathrm{dmit})_{2}\right]_{2}[7,8]$ (DMIT), and $\kappa-\mathrm{H}_{3}(\mathrm{Cat}-\mathrm{EDT}-\mathrm{TTF})_{2}$ [9-11] (H-Cat), and inorganic $\mathrm{YbMgGaO}_{4}$ [12-14]. In particular, in the above organic systems, no magnetic ordering occurs at least down to $1 / 1000$ of $J / k_{B}=200-300 \mathrm{~K}(J$ is the exchange interaction between neighboring spins) [5,7].

The most remarkable and intriguing feature in the QSL state on a triangular lattice is that in DMIT and H-Cat the heat capacity has a nonzero linear temperature term $\gamma T$ and thermal conductivity has a finite residual linear term $\kappa_{0} / T \equiv \kappa / T(T \rightarrow 0)[10,11,15,16]$. (Recent measurements report that there are two types of DMIT with and without finite $\kappa_{0} / T$ [17-19].) This demonstrates the emergence of gapless spin excitations that behave like mobile charge carriers in a paramagnetic metal with a Fermi surface, although the charge degrees of freedom are gapped. The gapless excitations has also been reported by magnetic susceptibility measurements [20]. The observed highly mobile and gapless excitations have been discussed in terms of fermionic spinons, fractionalized particles that carry spin but no charge, that form the Fermi surface $[21,22]$. On the other hand, the finite residual linear term $\kappa_{0} / T$ is not observed in thermal conductivity of BEDTTTF and $\mathrm{YbMgGaO}_{4}[6,23]$, even though the nonzero linear term $\gamma T$ is observed in heat capacity [12,24].

The effect of quenched disorder has been a fundamental and longstanding issue of the QSL ground states, which has been extensively addressed theoretically. Randomness had been considered to be detrimental for QSL states because it can localize the resonating singlet spins. In recent years, on the other hand, it has been pointed out that strong randomness can act as an additional frustration, which stabilizes QSL states [25]. Moreover, it has been suggested that even weaker bond randomness can affect quantum paramagnetic phases such as QSLs by inducing orphan spins, as emergent spin defects created by the surrounding valence bonds [26].

However, despite its importance, the systematic effect of randomness on the QSL ground states has been little explored experimentally because of the lack of appropriate systems. In the organic compounds, for example, the triangular lattice consisting of large molecular dimers with spin- $1 / 2$ is distorted from the perfect triangular lattice. Moreover, the glassy dielectric behavior observed in the organic salts suggests the charge imbalance within large molecular dimers, whose influence on the QSL state is unclear. In $\mathrm{YbMgGaO}_{4}$, $\mathrm{Mg}^{2+} / \mathrm{Ga}^{3+}$ site mixing introduces strong randomness in the exchange interaction between spin- $1 / 2$ on $\mathrm{Yb}$ sites. Moreover, in $\mathrm{YbMgGaO}_{4}$ with $J / k_{B} \sim 4 \mathrm{~K}$, low-energy excitations are difficult to extract at low enough temperature, $T \ll J / k_{B}$ [12-14]. The presence of randomness is also pointed out in QSL candidates on other model systems such as herbertsmithite $\mathrm{ZnCu}_{3}(\mathrm{OH})_{6} \mathrm{Cl}_{2}$, which consists of $\mathrm{Cu}$ kagome layers separated by nonmagnetic $\mathrm{Zn}$ layers. In this system, random substitution of magnetic $\mathrm{Cu}^{2+}$ by nonmagnetic $\mathrm{Zn}^{2+}$ (and vice versa) together with a possible Jahn-Teller distortion induces the effective quenched randomness [27]. Furthermore, in the above systems, degree of randomness cannot be controlled. Thus, the situation calls for a new system. (a)

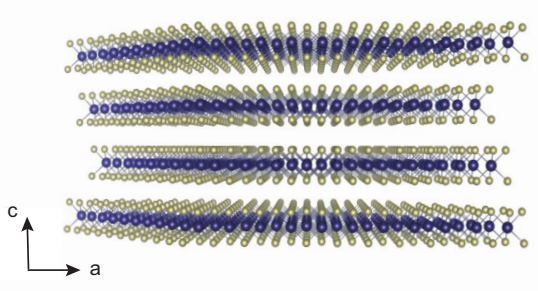

(b)

FIG. 1. (a) Crystal structure of $1 \mathrm{~T}-\mathrm{TaS}_{2}$. Each layer consists of tantalum atoms (purple) sandwiched by sulfur atoms (yellow). (b) A schematics of the star-of-David clusters, which appear as a result of the C-CDW transition. In the Mott insulating state, the electrons localized at the centers of the clusters form $S=1 / 22 \mathrm{D}$ perfect triangular lattice.

Recently, transition metal dichalcogenide $1 \mathrm{~T}-\mathrm{TaS}_{2}$ has aroused great interest as a candidate material that hosts a QSL ground state on the 2D perfect triangular lattice [28]. This compound is a layered material that contains one Ta layer sandwiched by two $\mathrm{S}$ layers; these layers are weakly coupled by van der Waals interactions, as illustrated in Fig. 1(a). The Ta atoms form a 2D triangular lattice. At high temperatures $(T>550 \mathrm{~K}), 1 \mathrm{~T}-\mathrm{TaS}_{2}$ is metallic. As the temperature is lowered, it exhibits an incommensurate CDW phase below $550 \mathrm{~K}$, followed by a nearly commensurate CDW (NC-CDW) phase below $350 \mathrm{~K}$. It undergoes a commensurate CDW (C-CDW) transition at $180 \mathrm{~K}$, below which the unit cell is reconstructed into a rotated triangular lattice characterized by $\sqrt{13} \times \sqrt{13}$ structure described as star-of-David clusters with 13 Ta atoms [29,30]. Strong electron correlation gives rise to a Mott insulating state, in which one localized electron resides at the center of the star-of-David cluster. As a result, a perfect 2D triangular lattice with $S=1 / 2$ is formed at low temperatures, as illustrated in Fig. 1(b). The nuclear magnetic resonance (NMR) experiments have reported the power law dependence of spin-lattice relaxation rate $1 / T_{1}$, indicating that the system is not in the band insulating state in which $1 / T_{1}$ decays exponentially. Muon spin relaxation and nuclear quadrupole resonance (NQR) experiments have reported no long-range magnetic ordering down to $20 \mathrm{mK}$, despite the strong exchange coupling $J / k_{B}$ which is at least hundreds of Kelvin as suggested by magnetic susceptibility measurements $[28,31]$. Moreover, gapless behavior of the spin dynamics has also been suggested by the muon and NQR measurements.

To reveal the nature of QSL states, it is crucially important to clarify whether the low-lying excitations are gapped or gapless and whether they are localized or itinerant. The heat capacity $C$ and thermal conductivity $\kappa$ measurements provide crucial information on these issues. The former includes both localized and itinerant excitations, while the latter sensitively probes elementary itinerant excitations and is totally insensitive to localized ones such as those responsible for Schottky contributions, which contaminates the heat capacity measurements at low temperatures. The $T$-linear term in the heat capacity $\gamma T$ has been reported, indicating the presence of gapless excitations [32]. The measurements of the thermal conductivity, on the other hand, has reported no discernible 
residual linear term in zero field, $\kappa_{0} / T=\kappa / T(T \rightarrow 0)=0$, indicating the absence of itinerant magnetic excitations [33]. Based on these results, the presence of the spinons that form a Fermi surface has been questioned.

In this paper, to obtain new insights into low-lying spin excitations of $1 \mathrm{~T}-\mathrm{TaS}_{2}$, the effect of randomness induced by quenched disorder on the spin liquid state is investigated. For this purpose, we performed systematic studies of lowtemperature heat capacity and thermal conductivity on pure, Se-substituted and electron-irradiated crystals of $1 \mathrm{~T}-\mathrm{TaS}_{2}$. Substitution of $\mathrm{S}$ by $\mathrm{Se}$ in the neighboring $\mathrm{S}$ layers induces the random exchange disorder in magnetic Ta layers, which would act as a weak disorder on the QSL. On the other hand, electron irradiation introduce atomic-scale point defects into Ta layers, which act as strong quenched disorder in $1 \mathrm{~T}-\mathrm{TaS}_{2}$. In fact, as shown later, a small number of vacancies at Ta site (two vacancies per $10^{5} \mathrm{Ta}$ atoms) dramatically influence the properties of QSL state. We report that the itinerant gapless excitations coexist with the localized gapless excitations. We find that the former excitations are strongly suppressed by the disorder, while the latter excitations are robust against the disorder. Moreover, the heat capacity arising from the latter excitations obeys a universal scaling relation, which assumes the presence of orphan spins forming random longrange valence bonds. Based on these results, we argue that localized orphan spins form random singlet valence bonds and are surrounded by a QSL phase with spinon Fermi surface. Moreover, introduction of strong disorder by electron irradiation leads to a possible new state of a spin liquid.

\section{SAMPLE PREPARATION AND METHODS}

High-quality $1 \mathrm{~T}-\mathrm{TaS}_{2}, 1 \mathrm{~T}-\mathrm{TaS}_{2-x} \mathrm{Se}_{x}(x=0.07$ and 0.57$)$ single crystals were grown by the chemical vapor transport method. To introduce spatially homogeneous strong disorder, we employed 2.5-MeV electron beam irradiation at the SIRIUS Pelletron linear accelerator operated by the Laboratorie des Solides Irradiées (LSI) at Ecole Polytechnique. This incident energy is sufficient to form vacancy-interstitial (Frenkel) pairs, which act as pointlike defects. With displacement energy of $10 \mathrm{eV}$, irradiation of $20 \mathrm{mC} / \mathrm{cm}^{2}$ dose causes $\approx 2$ vacancies per $10^{5} \mathrm{Ta}$ sites, which is about three times larger than the number of vacancies for $\mathrm{S}$ sites.

Figure 2 depicts the temperature dependence of the resistivity $\rho(T)$ for crystals of $1 \mathrm{~T}-\mathrm{TaS}_{2}$ (\#1 and \#2), $1 \mathrm{~T}-\mathrm{TaS}_{1.93} \mathrm{Se}_{0.07}, 1 \mathrm{~T}-\mathrm{TaS}_{1.43} \mathrm{Se}_{0.57}$, and electron-irradiated $1 \mathrm{~T}-\mathrm{TaS}_{2} \quad$ (Irr). $\quad$ For $1 \mathrm{~T}-\mathrm{TaS}_{2} \quad(\# 1), \quad 1 \mathrm{~T}-\mathrm{TaS}_{1.93} \mathrm{Se}_{0.07}$, $1 \mathrm{~T}-\mathrm{TaS}_{1.43} \mathrm{Se}_{0.57}$, and $1 \mathrm{~T}-\mathrm{TaS}_{2}$ (Irr), $\rho(T)$ was measured both on cooling and on warming. The hysteresis around $200 \mathrm{~K}$ is due to the first-order phase transition between NC-CDW and C-CDW [29,30]. The width of the hysteresis loop of the resistivity is enhanced in $1 \mathrm{~T}-\mathrm{TaS}_{1.43} \mathrm{Se}_{0.57}$ and electron-irradiated $1 \mathrm{~T}-\mathrm{TaS}_{2}$, indicating that the first-order phase transition is broadened by the disorder. All the crystals show the insulating behavior at low temperatures, but there is no clear trends in the amplitude of the resistivity on the defect levels. It has been suggested that stacking faults of $\mathrm{TaS}_{2}$ layers or remaining domain boundaries of NC-CDW give rise to the filamentary metallic conduction at low temperatures [34], although their contributions to the heat

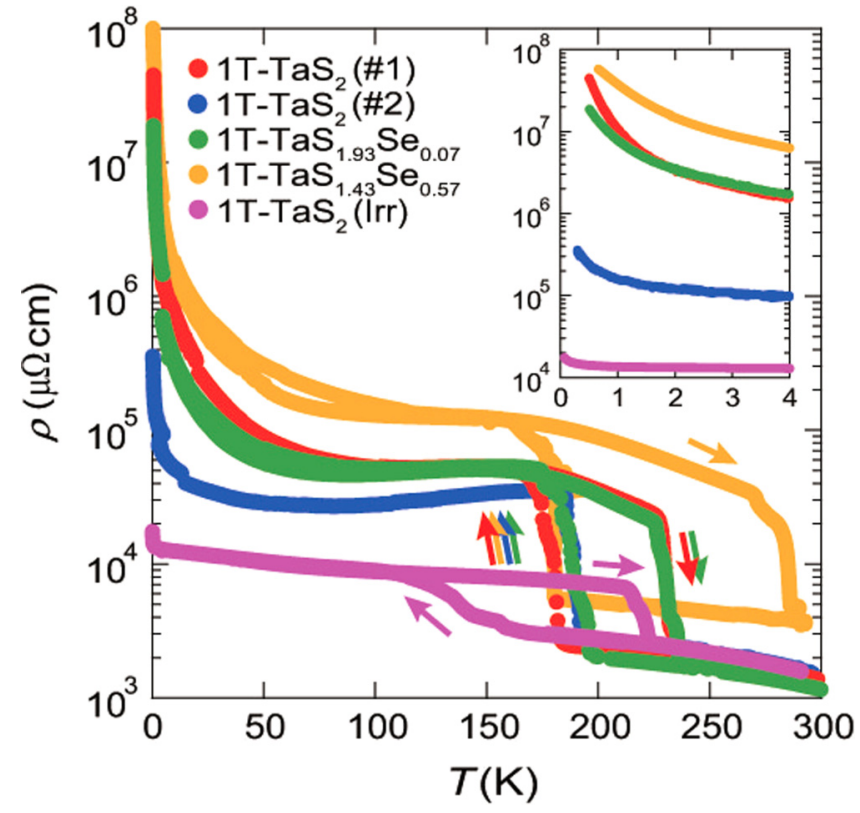

FIG. 2. Temperature dependences of the resistivity in single crystals of pure $1 \mathrm{~T}-\mathrm{TaS}_{2} \# 1$ (red), 1T- $\mathrm{TaS}_{2} \# 2$ (blue), $1 \mathrm{~T}-\mathrm{TaS}_{1.93} \mathrm{Se}_{0.07}$ (green), $1 \mathrm{~T}-\mathrm{TaS}_{1.43} \mathrm{Se}_{0.57}$ (orange), and electronirradiated $1 \mathrm{~T}-\mathrm{TaS}_{2}$ (Irr) (purple). The resistivity is measured for both cooling and heating processes except for $1 \mathrm{~T}-\mathrm{TaS}_{2}(\# 2)$. For $1 \mathrm{~T}-\mathrm{TaS}_{2}$ (\#2), data obtained for the cooling process is shown. The inset shows the resistivity below $4 \mathrm{~K}$.

capacity is negligibly small. Therefore, the magnitude of the resistivity at low temperature may not be directly related to the defect level. We also note that in $\mathrm{TaS}_{2}$ where the finite residual $T$-linear thermal conductivity observed, the Lorentz number $L=\left(\kappa_{0} / T\right) \rho$ estimated from $\kappa_{0} / T \sim 0.05 \mathrm{~W} / \mathrm{K}^{2} \mathrm{~m}$ and $\rho=10^{6}-10^{8} \mu \Omega \mathrm{cm}$ at the lowest temperature is $\approx 10^{4}-10^{6}$ times larger than that of a conventional metal $L_{0}=\pi^{2} / 3\left(k_{B} / e\right)^{2}=2.44 \times 10^{-8} \mathrm{~W} \Omega / \mathrm{K}^{2}$, indicating a spectacular violation of Wiedemann-Franz law. This shows that the filamentary metallic conduction is not relevant to the finite residual $T$-linear thermal conductivity.

The thermal conductivity was measured down to $80 \mathrm{mK}$ by the standard one-heater-two-thermometers steady-state technique. The crystal sizes of $1 \mathrm{~T}-\mathrm{TaS}_{2} \# 1$, $1 \mathrm{~T}-\mathrm{TaS}_{2} \# 2,1 \mathrm{~T}-\mathrm{TaS}_{1.93} \mathrm{Se}_{0.07}, 1 \mathrm{~T}-\mathrm{TaS}_{1.43} \mathrm{Se}_{0.57}$, and $1 \mathrm{~T}-\mathrm{TaS}_{2}$ (Irr) are 0.42 (distance between contacts) $\times 0.26$ (width) $\times 0.025$ (thickness), $0.98 \times 0.250 \times 0.042,1.7 \times 0.37 \times$ $0.080,0.81 \times 0.26 \times 0.073$, and $0.45 \times 0.76 \times 0.023 \mathrm{~mm}^{3}$, respectively. The heat current was applied parallel to the 2D plane and the magnetic field was applied perpendicular to the 2D plane. As shown in Fig. 2, the magnitude of the resistivity at low temperature is sample dependent. Therefore, it is crucially important to measure the thermal conductivity and the heat capacity on the same crystal. To measure the heat capacity of tiny crystals used for the thermal conductivity experiments, we used a long relaxation method [35]. For both measurements, the crystals were cooled down very slowly, as the first-order CDW transition is sensitive to the cooling rate and transition is smeared out by a rapid cooling. 


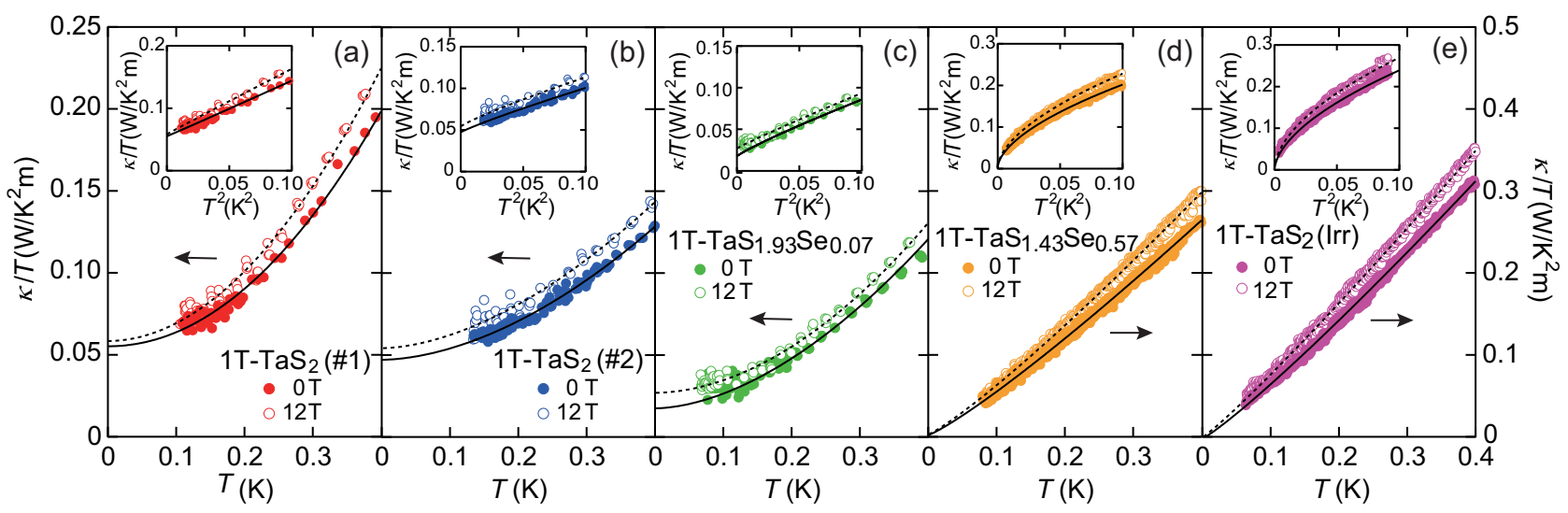

FIG. 3. Temperature dependences of $\kappa / T$ for (a) $1 \mathrm{~T}-\mathrm{TaS}_{2} \# 1$, (b) $1 \mathrm{~T}-\mathrm{TaS}_{2} \# 2$, (c) $1 \mathrm{~T}-\mathrm{TaS}_{1.93} \mathrm{Se}_{0.07}$, (d) $1 \mathrm{~T}-\mathrm{TaS}_{1.43} \mathrm{Se}_{0.57}$, and (e) $1 \mathrm{~T}-\mathrm{TaS}_{2}$ (Irr) in zero magnetic field (filled circles) and at $\mu_{0} H=12 \mathrm{~T}$ applied along $c$ axis (open circles). The insets show $\kappa / T$ plotted as a function of $T^{2}$. Solid and dotted lines in panels (a)-(c) show the fits by the formula $\kappa / T=\kappa_{0} / T+b_{1} T^{2}$ for 0 and $12 \mathrm{~T}$, respectively. Solid and dotted lines in panels (d) and (f) show the fits by $\kappa / T=\kappa_{0} / T+b_{2} T^{p}$ with $p \approx 1$ for $0 \mathrm{~T}$ and $12 \mathrm{~T}$, respectively. The residual value $\kappa_{0} / T\left(\approx 0.05 \mathrm{~W} / \mathrm{K}^{2} \mathrm{~m}\right)$ for $1 \mathrm{~T}-\mathrm{TaS}_{2} \# 1$ and \#2 is largely suppressed in $1 \mathrm{~T}-\mathrm{TaS}_{1.93} \mathrm{Se}_{0.07}\left(\kappa_{0} / T \approx 0.02 \mathrm{~W} / \mathrm{K}^{2} \mathrm{~m}\right)$. In $1 \mathrm{~T}-\mathrm{TaS}_{1.43} \mathrm{Se}_{0.57}$ and $1 \mathrm{~T}-\mathrm{TaS} \mathrm{S}_{2}(\mathrm{Irr}), \kappa_{0} / T$ is absent.

\section{RESULTS AND DISCUSSION}

\section{A. Thermal conductivity of $1 \mathrm{~T}-\mathrm{TaS}_{2}$}

Figures 3(a)-3(e) show the thermal conductivity divided by temperature $\kappa / T$ plotted as a function of temperature for $1 \mathrm{~T}-\mathrm{TaS}_{2} \# 1$, \#2, $1 \mathrm{~T}-\mathrm{TaS}_{1.93} \mathrm{Se}_{0.07}, 1 \mathrm{~T}-\mathrm{TaS}_{1.43} \mathrm{Se}_{0.57}$, and $1 \mathrm{~T}-\mathrm{TaS}_{2}$ (Irr), respectively, at low temperatures in zero field (filled circles) and at $\mu_{0} H=12 \mathrm{~T}$ (open circles) applied perpendicular to the $2 \mathrm{D}$ plane. The insets show the same data plotted as a function of $T^{2}$. As the temperature is lowered, $\kappa / T$ decreases in proportion to $T^{2}$. On the other hand, as shown in the main panels and insets of Figs. 3(d) and $3(\mathrm{e}), \kappa / T$ for $1 \mathrm{~T}-\mathrm{TaS}_{1.43} \mathrm{Se}_{0.57}$ and $1 \mathrm{~T}-\mathrm{TaS}_{2}$ (Irr) decreases as $\kappa / T \propto T^{p}$ with $p \approx 1$. The best fit is obtained by $p=$ 1.15 and $1.09-1.12$ for $1 \mathrm{~T}-\mathrm{TaS}_{1.43} \mathrm{Se}_{0.57}$ and $1 \mathrm{~T}-\mathrm{TaS}_{2}$ (Irr), respectively. In insulating magnets, thermal conductivity can be written as a sum of the spin and phonon contributions, $\kappa=$ $\kappa_{p h}+\kappa_{\text {spin }}$. When the mean free path of acoustic phonon $\ell_{p h}$ exceeds the average size of the samples at low temperature, the phonons undergo specular or diffuse scattering from a crystal boundary. The phonon conductivity in the boundary scattering regime is expressed as $\kappa_{p h}=\frac{1}{3} C_{p h}\left\langle v_{s}\right\rangle \ell_{p h}$, where $C_{p h}=\beta T^{3}$ is the phonon heat capacity of the Debye model with coefficient $\beta$ and $\left\langle v_{s}\right\rangle$ is the mean acoustic phonon velocity. For diffuse scattering limit due to rough surface roughness, $\ell_{p h}$ becomes $T$ independent, resulting in $\kappa_{p h} \propto T^{3}$. For specular reflection limit, on the other hand, $\ell_{p h}$ depends on $T$ as $\ell_{p h} \propto 1 / T$, leading to $\kappa_{p h} \propto T^{2}$. In real systems, the phonon conductivity depends on $T$ as $\kappa_{p h} \propto T^{\alpha}$ with $\alpha$ of intermediate value between 2 and 3. Thus, $1 \mathrm{~T}-\mathrm{TaS}_{2}$ (\#1 and \#2) and $1 \mathrm{~T}-\mathrm{TaS}_{1.93} \mathrm{Se}_{0.07}$ crystals are close to the diffuse scattering limit, while $1 \mathrm{~T}-\mathrm{TaS}_{1.43} \mathrm{Se}_{0.57}$ and electron-irradiated $1 \mathrm{~T}-\mathrm{TaS}_{2}$ crystals are close to specular reflection limit. These appear to be consistent with the fact that $\kappa_{p h}$ of $1 \mathrm{~T}-\mathrm{TaS}_{2}$ and $1 \mathrm{~T}-\mathrm{TaS}_{1.93} \mathrm{Se}_{0.07}$ is smaller than $\kappa_{p h}$ of $1 \mathrm{~T}-\mathrm{TaS}_{1.43} \mathrm{Se}_{0.57}$ and electron-irradiated $1 \mathrm{~T}-\mathrm{TaS}_{2}$, because $\ell_{p h}$ in the specular reflection limit can be enhanced from $\ell_{p h}$ in the diffuse scattering limit.
The finite intercept, shown by both plots of $\kappa / T$ versus $T$ and $\kappa / T$ versus $T^{2}$ depicted in Figs. 3(a)-3(c) and their insets, provides evidence for finite $\kappa_{0} / T$, demonstrating the presence of mobile and gapless spin excitations. This provides support on the presence of emergent spinons that form a Fermi surface $[21,22,36]$. We note that the observed finite $\kappa_{0} / T$ rules out a possibility of spin liquids with gapless Dirac-like spinons. The magnitude of $\kappa_{0} / T\left(\approx 0.05 \mathrm{~W} / \mathrm{K}^{2} \mathrm{~m}\right)$ for both $1 \mathrm{~T}-\mathrm{TaS}_{2} \# 1$ and \#2 is largely suppressed by Se substitution in $1 \mathrm{~T}-\mathrm{TaS}_{1.93} \mathrm{Se}_{0.07}\left(\kappa_{0} / T \approx 0.02 \mathrm{~W} / \mathrm{K}^{2} \mathrm{~m}\right)$. Moreover, as shown in Fig. 3(d), both plots of $\kappa / T$ versus $T$ and $\kappa / T$ versus $T^{2}$ show vanishing of $\kappa_{0} / T$ in $1 \mathrm{~T}-\mathrm{TaS}_{1.43} \mathrm{Se}_{0.57}$. These results indicate that even weak disorder introduced by Se substitution in the neighboring layers of magnetic Ta layers leads to a large suppression of conduction of the itinerant quasiparticles. In $1 \mathrm{~T}-\mathrm{TaS}_{2}$ (Irr), the strong disorder from irradiation also gives rise to vanishing $\kappa_{0} / T$. In an applied magnetic field, while $\kappa_{0} / T$ is almost unchanged within error bars, $\kappa / T$ is slightly enhanced at finite temperatures, which may be attributed to the enhancement of phonon mean free path due to the suppression of the spin-phonon scattering by external magnetic field.

The observed finite $\kappa_{0} / T$ in pure $1 \mathrm{~T}-\mathrm{TaS}_{2}$ is inconsistent with the previous results which report the absence of $\kappa_{0} / T$ [33]. The vanishing of $\kappa_{0} / T$ in $1 \mathrm{~T}-\mathrm{TaS}_{1.93} \mathrm{Se}_{0.07}$ indicates that this discrepancy may be due to the difference of the degree of disorder between the present crystals and the crystals used in Ref. [33]. The present results suggest that the crystals with a small amount of disorder may be required to observe finite $\kappa_{0} / T$.

\section{B. Heat capacity of 1T-TaS $\mathrm{T}_{2-x} \mathrm{Se}_{x}$}

Figures 4(a)-4(d) depict the heat capacity divided by temperature $C / T$ plotted as a function of $T^{2}$ in zero and finite magnetic fields applied perpendicular to the $2 \mathrm{D}$ plane for $1 \mathrm{~T}-\mathrm{TaS}_{2}$ (\#1, \#2), $1 \mathrm{~T}-\mathrm{TaS}_{1.43} \mathrm{Se}_{0.57}$, and $1 \mathrm{~T}-\mathrm{TaS}_{2}$ (Irr), respectively. In zero field, $C / T$ exhibits an upturn at low 


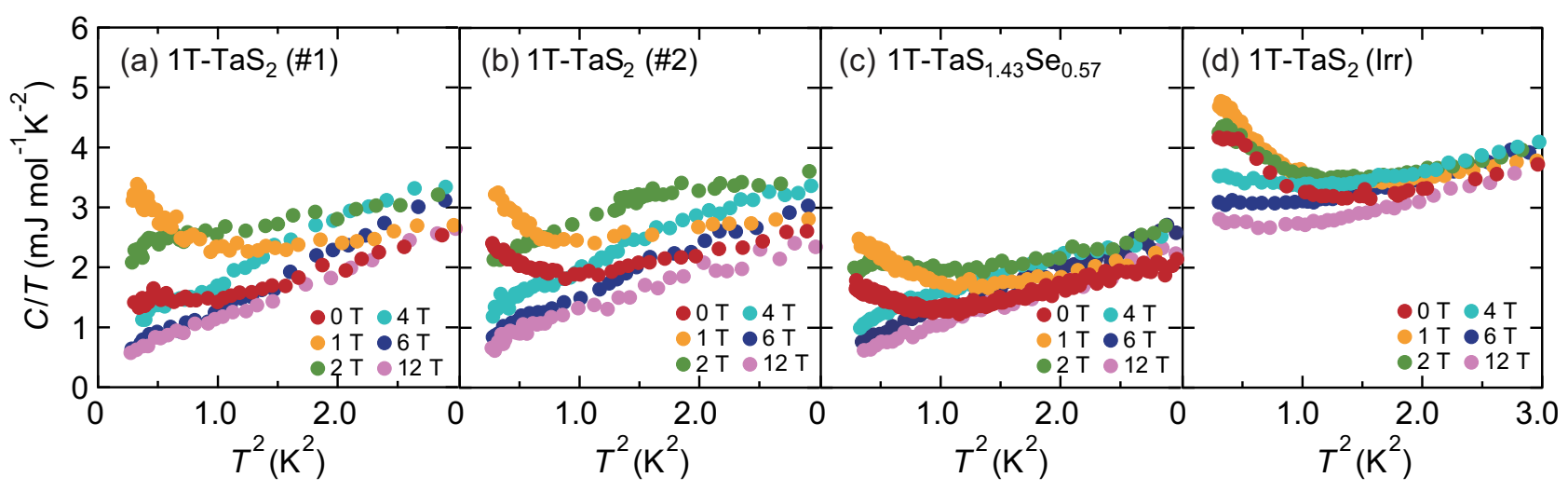

FIG. 4. $C / T$ plotted as a function of $T^{2}$ for (a) $1 \mathrm{~T}-\mathrm{TaS}_{2} \# 1$, (b) $1 \mathrm{~T}-\mathrm{TaS}_{2} \# 2$, (c) $1 \mathrm{~T}-\mathrm{TaS}_{1.43} \mathrm{Se}_{0.57}$, and (d) $1 \mathrm{~T}-\mathrm{TaS} \mathrm{S}_{2}$ (Irr) in zero field and at $\mu_{0} H=1,2,4,6$, and $12 \mathrm{~T}$. For all the samples, an upturn of $C / T$ is observed at low temperatures at low fields.

temperatures for all the samples. The upturn is enhanced at low fields, but it is largely suppressed at high fields. In the high-temperature regime, where no upturn is observed, $C / T$ increases in proportion to $T^{2}$. There are two important features that should be emphasized in the heat capacity. The first feature is that a simple extrapolation of $C / T$ to $T \rightarrow 0$ from high-temperature region above $1 \mathrm{~K}$ shows finite intercept at $T=0$, demonstrating the presence of a finite $\gamma T$ term that represents the gapless fermionic excitations. Thus $C / T$ is well fitted as $C / T=\gamma+\beta T^{2}$ in the temperature range where upturn of $C / T$ is not observed. For $1 \mathrm{~T}-\mathrm{TaS}_{2}$ (\#1 and \#2) and $1 \mathrm{~T}-\mathrm{TaS}_{1.43} \mathrm{Se}_{0.57}$, the upturn of $C / T$ at low temperatures is not observed at $\mu_{0} H \geqslant 4 \mathrm{~T}$ and $C / T=\gamma+\beta T^{2}$ holds in the whole temperature range. It is obvious that as shown in Fig. 4(a), $\gamma$ in zero field is largely enhanced at $\mu_{0} H=2 \mathrm{~T}$, while it is largely suppressed at $\mu_{0} H=12 \mathrm{~T}$.

In Fig. 5, the detailed field dependence of $\gamma(H)$ obtained by the extrapolation above $1 \mathrm{~K}$ is shown. For all crystals, $\gamma$ initially increases with $H$, and then peaks and decreases.

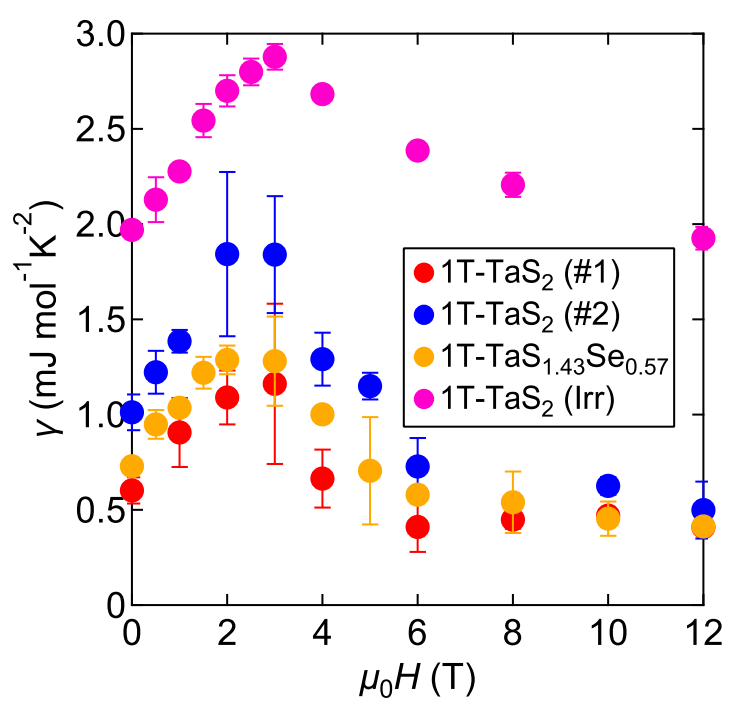

FIG. 5. Magnetic field dependences of $\gamma$ for $1 \mathrm{~T}-\mathrm{TaS}_{2} \# 1$ (red), $1 \mathrm{~T}-\mathrm{TaS}_{2} \# 2$ (blue), $1 \mathrm{~T}-\mathrm{TaS}_{1.43} \mathrm{Se}_{0.57}$ (orange), and $1 \mathrm{~T}-\mathrm{TaS} \mathrm{S}_{2}$ (Irr) (pink) obtained by the extrapolation above $1 \mathrm{~K}$. For all the systems, $\gamma$ increases with $H$ at low fields, peaks, and decreases at high fields.
In stark contrast to $\kappa / T$ which is only slightly enhanced by the magnetic field, $\gamma$ is remarkably suppressed by the field at $\mu_{0} H \geqslant 3 \mathrm{~T}$. The thermal conductivity is governed by the itinerant excitations, while the heat capacity contains both localized and itinerant excitations. Therefore, these highly unusual contrasting response to magnetic field firmly establishes the presence of two types of gapless excitations with itinerant and localized characters. In $1 \mathrm{~T}-\mathrm{TaS}_{1.43} \mathrm{Se}_{0.57}$ and $1 \mathrm{~T}-\mathrm{TaS}_{2}$ (Irr), $\gamma$ remains finite, while $\kappa_{0} / T$ is absent. These results indicate that the itinerant gapless excitations are sensitive to the disorder, while the localized gapless excitations are robust against the disorder.

The contrasting response of the field dependence to adding disorder therefore also indicates that the $\gamma$ term mainly arises from the localized spin excitations. Therefore, the $\gamma$ term should arise from some gapless localized excitations of spin singlets. However, as a magnetic field has no effect on the singlet energy but rather breaks a singlet and turns the pair of spins into a triplet, the resulting $\gamma$ term would then be expected to be suppressed by a magnetic field. Therefore, the low-field enhancement of the $\gamma$ term cannot be explained by a simple gapless localized spin-singlet model.

The second important feature is the the upturn of $C / T$ at low temperatures. There are two scenarios for this upturn. One is the nuclear Schottky contribution $C_{S}$ and the other is the contribution of orphan spins that form random long-range valence bonds. We first analyze the data in accordance with the two-level Schottky model, where the upturn is described by

$$
C_{S}=A\left[\frac{\Delta(H)}{k_{B} T}\right]^{2} e^{-\Delta(H) / k_{B} T}
$$

Here, $A$ is a constant that is proportional to the number of two-level systems and $\Delta(H)=g \mu_{B} \sqrt{H_{0}^{2}+H^{2}}$ is the energy of the excited level, where $H_{0}$ is the magnetic field that is characterized by the crystal electric field, $g$ is the electron $g$ factor, which is assumed as 2 here, and $\mu_{B}$ is the Bohr magneton. Figures 6(a)-6(d) depict the upturn component of the heat capacity at low temperature $C_{u} / T$, which is yielded by subtracting $\gamma$ and $\beta T^{2}$ from total heat capacity $C_{u} / T=$ $C / T-\gamma-\beta T^{2}$. Here $\gamma$ and $\beta T^{2}$ are obtained from $C / T$ above $1 \mathrm{~K}$, where the heat capacity is not influenced by the 


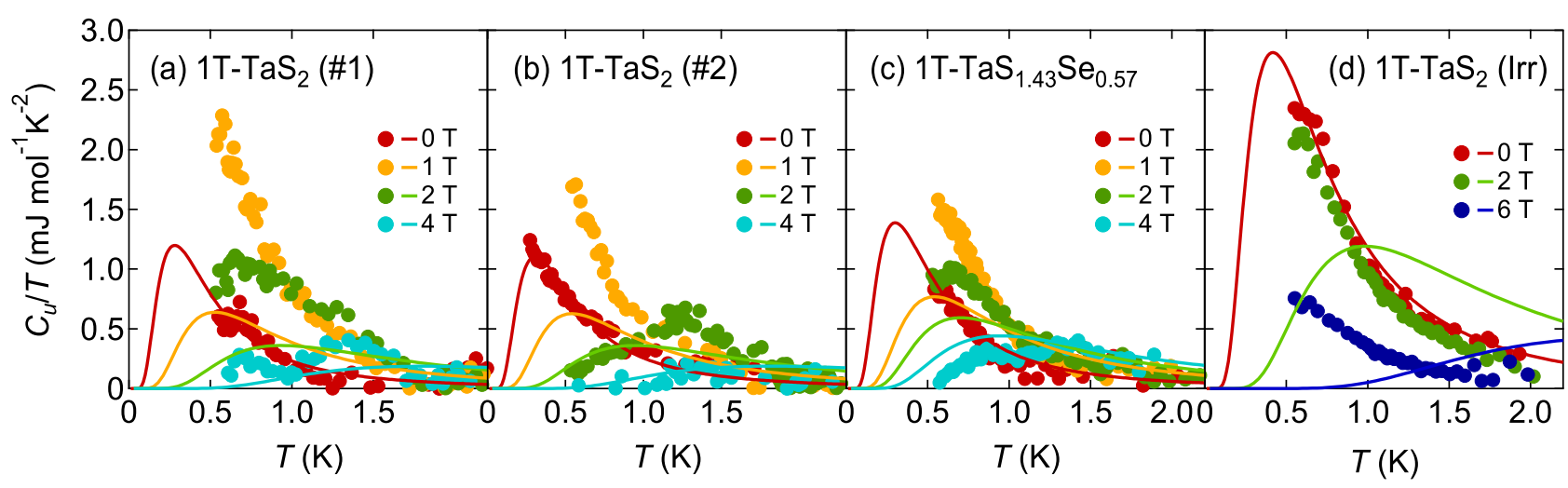

FIG. 6. Temperature dependence of $C_{u} / T=C / T-\gamma-\beta T^{2}$. Solid lines represent Schottky contribution $C_{S}$ calculated from Eq. (1). The parameters $A$ and $H_{0}$ in Eq. (1), both of which are field independent, are obtained from a fit to $C_{u} / T$ in zero field.

low-temperature upturn. We fit $C_{u} / T$ by Eq. (1) in zero field to determine $A$ and $H_{0}$, both of which are field independent, and then calculate $C_{S}$ in magnetic fields. As shown in Figs. 6(a)-6(d), $C_{u} / T$ in magnetic fields strongly deviates from the calculated $C_{S}$ (solid lines) for all the crystals. We find that in order to fit $C_{u} / T$ in the whole field range, we need to assume strongly field-dependent $A$, which is unphysical. Thus, the low-temperature upturn of $C / T$ is unlikely to be due to the Schottky contribution.

Thus, to account for the unusual field dependence of the $\gamma$ term and low-temperature enhancement of $C / T$, a new idea is required. Recently, through new theoretical studies of the role of quenched disorder in quantum paramagnetic states, an alternative scenario has been proposed [26,37]. In this picture, as shown schematically in Fig. 7, the majority of spin- $1 / 2$ sites form a quantum paramagnetic state such as a spin liquid, while a small fraction of sites host nucleated "orphan spins," which are not microscopic defects but rather are emergent quantum objects that arise from a competition of disorder

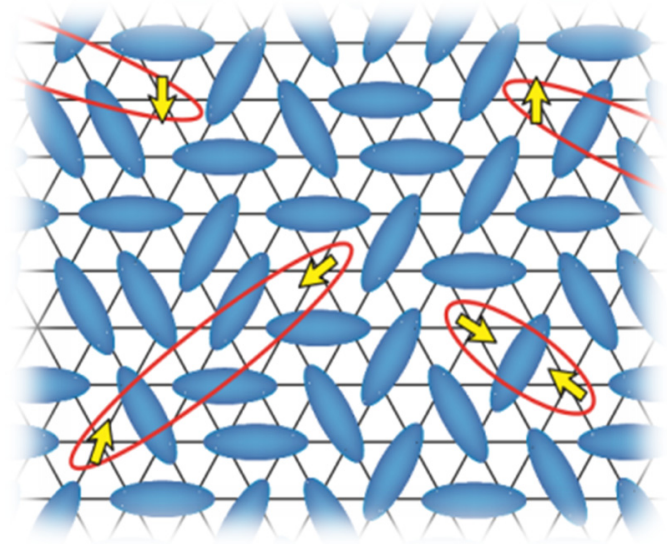

FIG. 7. A schematic illustration of the proposed spin liquid state of $1 \mathrm{~T}-\mathrm{TaS}_{2}$, which contains gapless excitations with localized and itinerant characters. The localized excitations arise from orphan spins (yellow arrows) that form random long-range valence bonds (red ellipsoids). They are surrounded by the proposed spin liquid state (blue ellipsoids) with itinerant excitations, which may be attributed to spinons that form a Fermi surface. and frustration. An orphan spin can couple with another orphan spin to form a singlet state. The exchange energies between these orphan spins vary randomly, depending on their distance, which leads to a formation of singlets with random energy gaps whose distribution is exponentially broad. This broad distribution includes singlets with arbitrarily small energy gaps (red ellipsoids in Fig. 7), leading to a state with gapless excitations.

These gapless excitations give rise to $\gamma$-term-like contributions in the heat capacity but similarly also contributes non- $T$-linear terms due to the nontrivial energy distribution of the random singlets. Combined, these contributions give rise to an enhancement of $C / T$ at low temperatures, in a way similar to the Schottky contribution but with a distinct field dependence. In particular, this model predicts that magnetic contribution $C_{m}$ in the heat capacity arising from the localized spin excitations collapse into a single curve of form,

$$
\frac{C_{m}(H, T)}{T} \sim \frac{1}{H^{\eta}} F_{q}(T / H),
$$

where $F_{q}(X)$ is a scaling function which is determined by the energy distribution of the random singlets:

$$
F_{q}(X) \sim \begin{cases}X^{q} & X \ll 1 \\ X^{-\eta}\left(1+c_{0} / X^{2}\right) & X \gg 1 .\end{cases}
$$

Here $\eta$ is a nonuniversal exponent, $0 \leqslant \eta \leqslant 1$, that characterizes the random-singlet distribution, $q$ is determined by the spin and crystal symmetries with $q=0$ for $1 \mathrm{~T}-\mathrm{TaS}_{2}$, and $c_{0}$ is a constant. When $\eta \neq 0, F_{0}(X)$ increases with $X$, peaks, and decreases at large $X$.

Here we analyze the heat capacity in accordance with the random long-range valence bonds scenario. To examine the scaling relation given by Eq. (2), the magnetic part is obtained by subtracting the acoustic phonon contribution, $C_{s p i n}=C-\beta T^{3}$. Here we use $\beta$ determined in zero field. Figures 8(a)-8(d) depicts the temperature dependence of $C_{\text {spin }} / T$. For $1 \mathrm{~T}-\mathrm{TaS}_{2}(\# 1, \# 2)$ and $1 \mathrm{~T}-\mathrm{TaS}_{1.43} \mathrm{Se}_{0.57}, C_{\text {spin }} / T$ increases with decreasing temperature at $\mu_{0} H \geqslant 2 \mathrm{~T}$ and is almost $T$ independent at the lowest temperature at $\mu_{0} H \geqslant 4 \mathrm{~T}$. On the other hand, in $1 \mathrm{~T}-\mathrm{TaS}_{2}$ (Irr), $C_{\text {spin }} / T$ shows an upturn in the whole field range.

In Figs. 9(a)-9(d), $C_{\text {spin }}\left(\mu_{0} \mathrm{Hr}\right)^{\eta} / \mathrm{T}$ is plotted as a function of $T / H$ for $1 \mathrm{~T}-\mathrm{TaS}_{2} \# 1, \# 2,1 \mathrm{~T}-\mathrm{TaS}_{1.43} \mathrm{Se}_{0.57}$, and $1 \mathrm{~T}-\mathrm{TaS}_{2}$ 


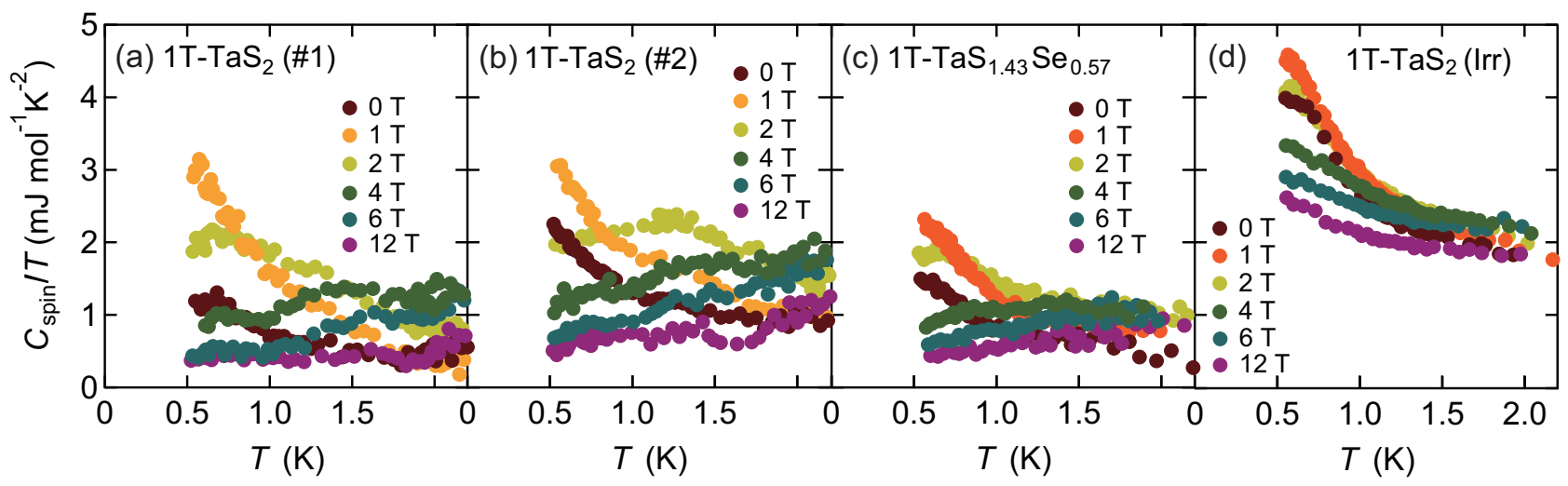

FIG. 8. Temperature dependence of $C_{\text {spin }} / T$ for (a) $1 \mathrm{~T}-\mathrm{TaS}_{2} \# 1$, (b) $1 \mathrm{~T}-\mathrm{TaS}_{2} \# 2$, (c) $1 \mathrm{~T}-\mathrm{TaS}_{1.43} \mathrm{Se}_{0.57}$, and (d) $1 \mathrm{~T}-\mathrm{TaS}$ (Irr) in zero field and at $\mu_{0} H=1,2,4,6$, and $12 \mathrm{~T}$.

(Irr), respectively, where $r=g / g_{0}$ with $g_{0}=2$ and the effective $g$ factor of $1 \mathrm{~T}-\mathrm{TaS}_{2}, g$. The fitting parameters are $\eta$ and $r$. We find that most data collapse into a single curve with $\eta=$ 0.68 and $r=1.37$ for $1 \mathrm{~T}-\mathrm{TaS}_{2} \# 1, \eta=0.44$ and $r=1.49$ for $1 \mathrm{~T}-\mathrm{TaS}_{2} \# 2$, and $\eta=0.49$ and $r=1.22$ for $1 \mathrm{~T}-\mathrm{TaS}_{1.43} \mathrm{Se}_{0.57}$. Deviation from the scaling function, which is observed at high fields $(H \geqslant 10 \mathrm{~T})$ and at the lowest field measured $(0.5 \mathrm{~T})$, can be ascribed to interplay of the local moments with the proposed spin liquid [37] and also to an error in estimating $\beta\left(\sim \pm 0.1 \mathrm{~mJ} / \mathrm{mol} \mathrm{K}^{4}\right)$. The scaling function obtained by Eq. (2) is shown by gray lines. Good scaling relation with using similar values of $\eta$ and $r$ suggest that three systems are in similar QSL states. These results, along with the presence of finite $\kappa_{0} / T$, suggest the microscopic coexistence of localized orphan spins that form random singlets and itinerant spinons that form Fermi surface. We note that the scaling function Eq. (2) assume only the localized excitations. Therefore, the scaling collapse shown in Figs. 9(a)-9(c) implies that the gapless excitations observed in the heat capacity are dominated by localized ones, i.e., $C_{s p i n} \approx C_{m}$. Small contribution of the itinerant gapless quasiparticles to the heat capacity would reflect a large exchange energy scale in $1 \mathrm{~T}-\mathrm{TaS}_{2}[36]$.
Let us then estimate the mean free path of the itinerant spinons. The thermal conductivity is written as $\kappa / T=$ $\frac{1}{3} \gamma_{s} v_{s} \ell_{s}$, where $\gamma_{s}$ is the heat capacity coefficient, $v_{s}$ is the velocity, and $\ell_{s}$ are the mean free path of the itinerant quasiparticles. Since gapless localized excitations dominate over gapless itinerant ones, $\gamma_{s} \ll \gamma$, the lower limit of $\ell_{s}$ is obtained. Using $v_{s} \sim \frac{J}{h}$, where $J \sim 1500 \mathrm{~K}$ is the exchange energy of spins [31] and $\gamma \sim 0.4 \mathrm{~mJ} / \mathrm{K}^{2} \mathrm{~mol}$ at $\mu_{0} H=12 \mathrm{~T}$ (this corresponds to $\approx 5.2 \mathrm{~mJ} / \mathrm{K}^{2}$ per spin), we obtain $\ell_{s} \gg$ $5 \mathrm{~nm}$, which is much longer than the interspin distance $(1 \mathrm{~nm})$. This long mean free path suggests that the itinerant spinons may be little scattered by the localized orphan spins, which deserves future study. This long mean free path is also consistent with the result that $\kappa_{0} / T$ is sensitive to Se substitution.

As shown in Fig. 9(d), although all data collapse into a single curve in $1 \mathrm{~T}-\mathrm{TaS}_{2}$ (Irr), the scaling function monotonically decreases with $T / H$, which is essentially different from that of $1 \mathrm{~T}-\mathrm{TaS}_{2}\left(\# 1\right.$ and \#2) and $1 \mathrm{~T}-\mathrm{TaS}_{1.43} \mathrm{Se}_{0.57}$. This scaling function cannot be described by Eqs. (2) and (3), as $F(X)$ increases with $X$ at low $X$. These results suggest that the strong disorder introduced in the magnetic Ta layers leads to a possible quantum paramagnetic state with an unusual scaling function.

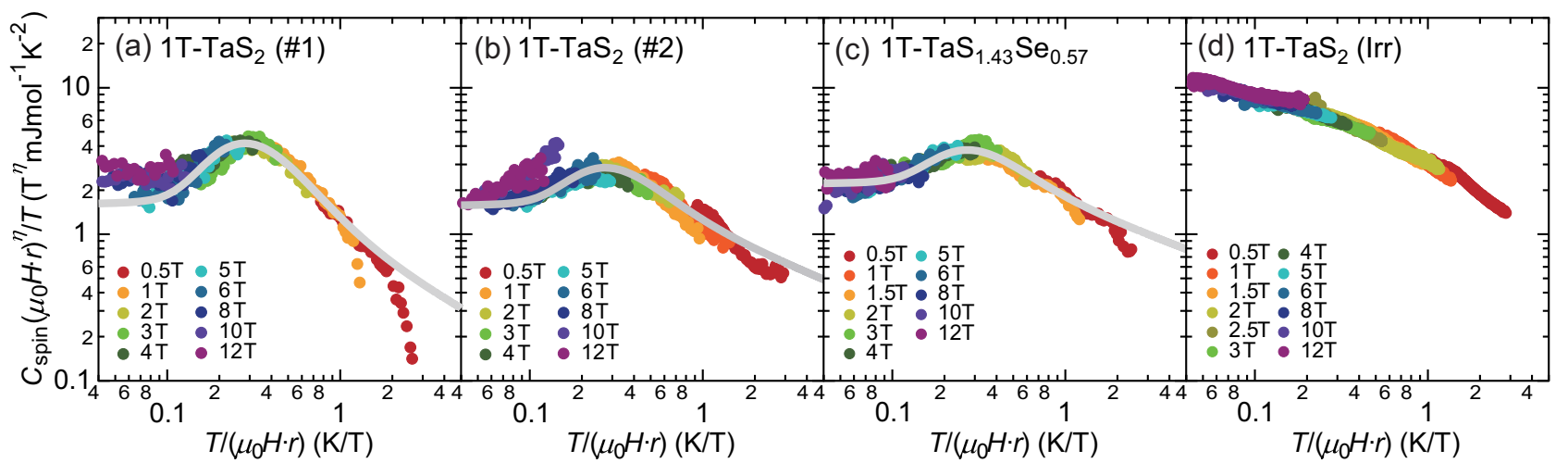

FIG. 9. Scaling relationship of spin contribution of the heat capacity; $C_{s p i n}\left(\mu_{0} H r\right)^{\eta} / T$ plotted as a function of $T /\left(\mu_{0} H r\right)$ for (a) $1 \mathrm{~T}-\mathrm{TaS} \mathrm{S}_{2}$ $\# 1$, (b) $1 \mathrm{~T}-\mathrm{TaS}_{2} \# 2$, (c) $1 \mathrm{~T}-\mathrm{TaS}_{1.43} \mathrm{Se}_{0.57}$, and (d) $1 \mathrm{~T}-\mathrm{TaS}_{2}$ (Irr). Good scaling is observed with the parameters of $\eta=0.68, r=1.37$ for $1 \mathrm{~T}-\mathrm{TaS}{ }_{2}$ $\# 1 ; \eta=0.44, r=1.49$ for $1 \mathrm{~T}-\mathrm{TaS}_{2} \# 2$; and $\eta=0.49, r=1.22$ for $1 \mathrm{~T}-\mathrm{TaS}_{1.43} \mathrm{Se}_{0.57}$. Gray lines for panels (a)-(c) indicate the scaling function obtained by the fit to Eq. (2). For $1 \mathrm{~T}-\mathrm{TaS}_{2}$ (Irr), although all the data collapse into a single curve, $C_{s p i n}\left(\mu_{0} H r\right)^{\eta} / T$ monotonically decreases with $T /\left(\mu_{0} H r\right)$ and cannot be described by Eqs. (2) and (3). 


\section{CONCLUSION}

To reveal the nature of the QSL state in $1 \mathrm{~T}-\mathrm{TaS}_{2}$ with the $2 \mathrm{D}$ perfect triangular lattice, we investigate the effect of randomness due to quenched disorder through the systematic measurements of heat capacity and thermal conductivity in pure, Se-substituted, and electron-irradiated crystals. The finite residual linear term of the thermal conductivity $\kappa_{0} / T$ indicates the presence of gapless itinerant quasiparticle excitations, which is consistent with the fractionalized spinons that form a Fermi surface. The conduction of this gapless itinerant quasiparticles is strongly suppressed by weak disorder. The finite-temperature linear heat capacity coefficient $\gamma$ is also observed. This $\gamma$ is robust against the disorder and exhibits very different field dependence than $\kappa_{0} / T$, demonstrating the coexistence of gapless excitations with itinerant and localized character. We find the universal scaling collapse of magnetic contribution of the heat capacity, which provides support that localized orphan spins form random singlets. These results appear to capture an essential feature of the QSL state of $1 \mathrm{~T}-\mathrm{TaS}_{2}$; localized orphan spins induced by disorder form random valence bonds and are surrounded by a QSL phase with the spinon Fermi surface. This feature is preserved by the introduction of weak quenched disorder by Se substitution. The introduction of strong disorder by electron irradiation, on the other hand, dramatically changes the spin liquid state, leading to a possible new quantum paramagnetic state. These unique features of $1 \mathrm{~T}-\mathrm{TaS}_{2}$ provide insights into the influence of quenched disorder on a QSL and demonstrate the ability to introduce distinct types of disorder with qualitatively different effects on QSL states.

\section{ACKNOWLEDGMENTS}

We are very grateful to H. Kawamura, K. T. Law, P.A. Lee, E.-G. Moon, K. Totsuka, and M. Udagawa for many useful comments and suggestions. This work was supported by Grants-in-Aid for Scientific Research (KAKENHI) (No. 25000003, No. 18H01177, No. 18H01180, No. 18H05227, and No. 18K13511), Innovative Areas Topological Material Science (No. JP15H05852), Quantum Liquid Crystals (No. JP19H05824), and 3D Active-Site Science (No. 26105004) from Japan Society for the Promotion of Science (JPSJ). I.K. was supported by a National Research Council Fellowship through the National Institute of Standards and Technology.
[1] L. Savary and L. Balents, Quantum spin liquids, Rep. Prog. Phys. 80, 016502 (2017).

[2] Y. Zhou, K. Kanoda, and T.-K. Ng, Quantum spin liquid states, Rev. Mod. Phys. 89, 025003 (2017).

[3] P. W. Anderson, Resonating valence bonds: A new kind of insulator? Mater. Res. Bull. 8, 153 (1973).

[4] P. Fazekas and P. W. Anderson, On the ground state properties of the anisotropic triangular antiferromagnet, Philos. Mag. 30, 423 (1974).

[5] Y. Shimizu, K. Miyagawa, K. Kanoda, M. Maesato, and G. Saito, Spin Liquid State in an Organic Mott Insulator with a Triangular Lattice, Phys. Rev. Lett. 91, 107001 (2003).

[6] M. Yamashita, N. Nakata, Y. Kasahara, T. Sasaki, N. Yoneyama, N. Kobayashi, S. Fujimoto, T. Shibauchi, and Y. Matsuda, Thermal-transport measurements in a quantum spin-liquid state of the frustrated triangular magnet $\kappa$-(BEDT-TTF $)_{2} \mathrm{Cu}_{2}(\mathrm{CN})_{3}$, Nat. Phys. 5, 44 (2009).

[7] T. Itou, A. Oyamada, S. Maegawa, M. Tamura, and R. Kato, Quantum spin liquid in the spin-1/2 triangular antiferromagnet $\mathrm{EtMe}_{3} \mathrm{Sb}\left[\mathrm{Pd}(\mathrm{dmit})_{2}\right]_{2}$, Phys. Rev. B 77, 104413 (2008).

[8] T. Itou, A. Oyamada, S. Maegawa, and R. Kato, Instability of a quantum spin liquid in an organic triangular-lattice antiferromagnet, Nat. Phys. 6, 673 (2010).

[9] T. Isono, H. Kamo, A. Ueda, K. Takahashi, M. Kimata, H. Tajima, S. Tsuchiya, T. Terashima, S. Uji, and H. Mori, Gappless Quantum Spin Liquid in an Organic Spin-1/2 TriangularLattice $\kappa-\mathrm{H}_{3}(\text { Cat-EDT-TTF })_{2}$, Phys. Rev. Lett. 112, 177201 (2014).

[10] M. Shimozawa, K. Hashimoto, A. Ueda, Y. Suzuki, K. Sugii, S. Yamada, Y. Imai, R. Kobayashi, K. Itoh, S. Iguchi, M. Naka, S. Ishihara, H. Mori, T. Sasaki, and M. Yamashita, Quantumdisordered state of magnetic and electric dipoles in an organic Mott system, Nat. Commun. 8, 1821 (2017).
[11] S. Yamashita, Y. Nakazawa, A. Ueda, and H. Mori, Thermodynamics of the quantum spin liquid state of the single-component dimer Mott system $\kappa-\mathrm{H}_{3}(\text { Cat-EDT-TTF })_{2}$, Phys. Rev. B 95, 184425 (2017).

[12] Y. Li, H. Liao, Z. Zhang, S. Li, F. Jin, L. Ling, L. Zhang, Y. Zou, L. Pi, Z. Yang, J. Wang, Z. Wu, and Q. Zhang, Gapless quantum spin liquid ground state in the two dimensional spin$1 / 2$ triangular antiferromagnet $\mathrm{YbMgGaO}_{4}$, Sci. Rep. 5, 16419 (2015).

[13] Y. Li, G. Chen, W. Tong, L. Pi, J. Liu, Z. Yang, X. Wang, and Q. Zhang, Rare-Earth Triangular Lattice Spin Liquid: A SingleCrystal Study of $\mathrm{YbMgGaO}_{4}$, Phys. Rev. Lett. 115, 167203 (2015).

[14] Y. Shen, Y. Li, H. Wo, Y. Li, S. Shen, B. Pan, Q. Wang, H. C. Walker, P. Steffens, M. Boehm, Y. Hao, D. L. Quintero-castro, L. W. Harriger, M. D. Frontzek, L. Hao, S. Meng, Q. Zhang, G. Chen, and J. Zhao, Evidence for a spinon Fermi surface in a triangular lattice quantum-spin-liquid candidate, Nature (London) 540, 559 (2016).

[15] M. Yamashita, N. Nakata, Y. Senshu, M. Nagata, H. M. Yamamoto, R. Kato, T. Shibauchi, and Y. Matsuda, Highly mobile gapless excitations in a two-dimensional candidate quantum spin liquid, Science 328, 1246 (2010).

[16] S. Yamashita, T. Yamamoto, Y. Nakazawa, M. Tamura, and R. Kato, Gapless spin liquid of an organic triangular compound evidenced by thermodynamic measurements, Nat. Commun. $\mathbf{2}$, 275 (2011).

[17] P. Bourgeois-Hope, F. Laliberté, E. Lefrançois, G. Grissonnanche, S. René de Cotret, R. Gordon, S. Kitou, H. Sawa, H. Cui, R. Kato, L. Taillefer, and N. Doiron-Leyraud, Thermal Conductivity of the Quantum Spin Liquid Candidate $\mathrm{EtMe}_{3} \mathrm{Sb}\left[\mathrm{Pd}(\mathrm{dmit})_{2}\right]_{2}$ : No Evidence of Mobile Gapless Excitations, Phys. Rev. X 9, 041051 (2019). 
[18] J. M. Ni, B. L. Pan, B. Q. Song, Y. Y. Huang, J. Y. Zeng, Y. J. Yu, E. J. Cheng, L. S. Wang, D. Z. Dai, R. Kato, and S. Y. $\mathrm{Li}$, Absence of Magnetic Thermal Conductivity in the Quantum Spin Liquid Candidate $\mathrm{EtMe}_{3} \mathrm{Sb}\left[\mathrm{Pd}(\mathrm{dmit})_{2}\right]_{2}$, Phys. Rev. Lett. 123, 247204 (2019).

[19] M. Yamashita, Boundary-limited and glassy-like phonon thermal conduction in $\mathrm{EtMe}_{3} \mathrm{Sb}\left[\mathrm{Pd}(\mathrm{dmit})_{2}\right]_{2}$, J. Phys. Soc. Jpn. 88, 083702 (2019).

[20] D. Watanabe, M. Yamashita, S. Tonegawa, Y. Oshima, H. M. Yamamoto, R. Kato, I. Sheikin, K. Behnia, T. Terashima, S. Uji, T. Shibauchi, and Y. Matsuda, Novel Pauli-paramagnetic quantum phase in a Mott insulator, Nat. Commun. 3, 1090 (2012).

[21] O. I. Motrunich, Variational study of triangular lattice spin$1 / 2$ model with ring exchanges and spin liquid state in $\kappa$ $(\mathrm{ET})_{2} \mathrm{Cu}_{2}(\mathrm{CN})_{3}$, Phys. Rev. B 72, 045105 (2005).

[22] S.-S. Lee and P. A. Lee, U(1) Gauge Theory of the Hubbard Model: Spin Liquid States and Possible Application to $\kappa$-(BEDT-TTF $)_{2} \mathrm{Cu}_{2}(\mathrm{CN})_{3}$, Phys. Rev. Lett. 95, 036403 (2005).

[23] Y. Xu, J. Zhang, Y. S. Li, Y. J. Yu, X. C. Hong, Q. M. Zhang, and S. Y. Li, Absence of Magnetic Thermal Conductivity in the Quantum Spin-Liquid Candidate $\mathrm{YbMgGaO}_{4}$, Phys. Rev. Lett. 117, 267202 (2016).

[24] S. Yamashita, Y. Nakazawa, M. Oguni, Y. Oshima, H. Nojiri, Y. Shimizu, K. Miyagawa, and K. Kanoda, Thermodynamic properties of a spin-1/2 spin-liquid state in a $\kappa$-type organic salt, Nat. Phys. 4, 459 (2008).

[25] K. Watanabe, H. Kawamura, H. Nakano, and T. Sakai, Quantum spin-liquid behavior in the spin-1/2 random Heisenberg antiferromagnet on the triangular lattice, J. Phys. Soc. Jpn. 83, 034714 (2014).

[26] I. Kimchi, A. Nahum, and T. Senthil, Valence Bonds in Random Quantum Magnets: Theory and Application to $\mathrm{YbMgGaO}_{4}$, Phys. Rev. X 8, 031028 (2018).

[27] J. S. Helton, K. Matan, M. P. Shores, E. A. Nytko, B. M. Bartlett, Y. Yoshida, Y. Takano, A. Suslov, Y. Qiu, J.-H. Chung, D. G. Nocera, and Y. S. Lee, Spin Dynamics of the Spin-1/2
Kagome Lattice Antiferromagnet $\mathrm{ZnCu}_{3}(\mathrm{OH})_{6} \mathrm{Cl}_{2}$, Phys. Rev. Lett. 98, 107204 (2007).

[28] K. T. Law and P. A. Lee, $1 \mathrm{~T}-\mathrm{TaS}_{2}$ as a quantum spin liquid, Proc. Natl. Acad. Sci. USA 114, 6996 (2017).

[29] K. Rossnagel, On the origin of charge-density waves in select layered transition-metal dichalcogenides, J. Phys.: Condens. Matter 23, 213001 (2011).

[30] M. Kratochvilova, A. D. Hillier, A. R. Wildes, L. Wang, S. Cheong, and J. Park, The low-temperature highly correlated quantum phase in the charge-density-wave $1 \mathrm{~T}-\mathrm{TaS}_{2}$ compound, npj Quantum Mat. 2, 42 (2017).

[31] M. Klanjšek, A. Zorko, R. Žitko, J. Mravlje, Z. Jagličić, P. K. Biswas, P. Prelovšek, D. Mihailovic, and D. Arčon, A hightemperature quantum spin liquid with polaron spins, Nat. Phys. 13, 1130 (2017).

[32] A. Ribak, I. Silber, C. Baines, K. Chashka, Z. Salman, Y. Dagan, and A. Kanigel, Gapless excitations in the ground state of 1T-TaS 2 , Phys. Rev. B 96, 195131 (2017).

[33] Y. J. Yu, Y. Xu, L. P. He, M. Kratochvilova, Y. Y. Huang, J. M. Ni, L. Wang, S.-W. Cheong, J.-G. Park, and S. Y. Li, Heat transport study of the spin liquid candidate $1 \mathrm{~T}-\mathrm{TaS}_{2}$, Phys. Rev. B 96, 081111(R) (2017).

[34] F. Zwick, H. Berger, I. Vobornik, G. Margaritondo, L. Forró, C. Beeli, M. Onellion, G. Panaccione, A. Taleb-Ibrahimi, and M. Grioni, Spectral Consequences of Broken Phase Coherence in 1T-TaS 2 , Phys. Rev. Lett. 81, 1058 (1998).

[35] Y. Kasahara, T. Ohnishi, Y. Mizukami, O. Tanaka, Sixiao Ma, K. Sugii, N. Kurita, H. Tanaka, J. Nasu, Y. Motome, T. Shibauchi, and Y. Matsuda, Majorana quantization and halfinteger thermal quantum Hall effect in a Kitaev spin liquid, Nature (London) 559, 227 (2018).

[36] W.-Y. He, X. Y. Xu, G. Chen, K. T. Law, and P. A. Lee, Spinon Fermi Surface in a Cluster Mott Insulator Model on a Triangular Lattice and Possible Application to $1 \mathrm{~T}-\mathrm{TaS}_{2}$, Phys. Rev. Lett. 121, 046401 (2018).

[37] I. Kimchi, J. P. Sheckelton, T. M. McQueen, and P. A. Lee, Scaling and data collapse from local moments in frustrated disordered quantum spin systems, Nat. Commun. 9, 4367 (2018). 